\title{
Remarks on the Method for Measuring Ion Beam Profiles
}

\author{
HoKotomo INOUYE* AND KenICHI NIURAO*
}

(Received 11 September 1972)

\begin{abstract}
Some conditions necessary for correctly measuring ion beam profiles by a transverse electric field are described. The most important is uniformity of the field. Gain uniformity on the first dynode surface of an elec tron multiplier used as the detector is also important. Fringing field effect can be safely ignored. The way of deducing the original profile by deconvolution of the measured intensity distribution curve proved to be satis. factorily accurate.
\end{abstract}

Accurate measurement of an ion beam profile is a procedure indispensable to obtaining correct information in the studies of sputtering, plasma phenomena, scattering, etc. Various methods for measuring beam profiles based on different principles have been published by several workers. ${ }^{1-7}$ ) The method described by Inouye and coworkers $^{3)}$ is different from the others in that it uses deflection of the beam by a transverse electric field. Their method is rather simple, although its application is limited to the charged particle beams. The method (hereafter, method I) consists of the following two procedures.

Procedure (1) After deflecting an ion beam by an electric field $E_{\mathrm{d}}$ perpendicular to the beam direction, the intensity $I(X)$ of the ion beam that passes through a detector hole with a diameter $\left(2 r_{0}\right)$ comparable to the beam diameter is measured. For the sake of convenience, $E_{\mathrm{d}}$ is usually assumed to be approximated by the applied voltage $V_{\mathrm{d}}$ divided by the electrode separation $d$. Thus, the displacement $X$ of the beam center is determined by Eqs. (1) and (2).

$$
X=(1 / 2)\left(E_{\mathrm{d}} / V_{\mathrm{a}}\right) l[L-(l / 2)],
$$

and

$$
E_{\mathrm{d}}=V_{d} / d \text {. }
$$

Figure 1 should be referred to for the symbols.

Procedure (2) The original beam profile is determined by deconvolution of the apparent intensity distribution curve $I(X)$. The beam profile is assumed to be a trapezoid with the base $2 D$ and the plateau $2 B$. Common volume $V(X)$ between a truncated cone and a cylinder ( $2 r_{0}$ in diameter) is expressed as a function of the interaxial distance $X$ for a set of $B$ and $D$ as parameters (see Fig. 2).

$$
V(X)=\int_{S(X)} I(R) d S,
$$

where $I(R)$ is the height of the truncated cone representing the ion beam intensity, $R$ the distance from the center of the beam, $S(X)$ the area of the base of the common solid and $d S$ the surface element of $S(X)$. The values of $B$ and $D$ for the $V(X)$ curve that best fit the $I(X)$ curve, which is normalized with respect to the value

\footnotetext{
* Research Institute for Scientific Measurements, Tohoku University (19-1. Sanjo-machi. Sendai. Japan).
} 


\section{H. Inouye and K. Niurao}

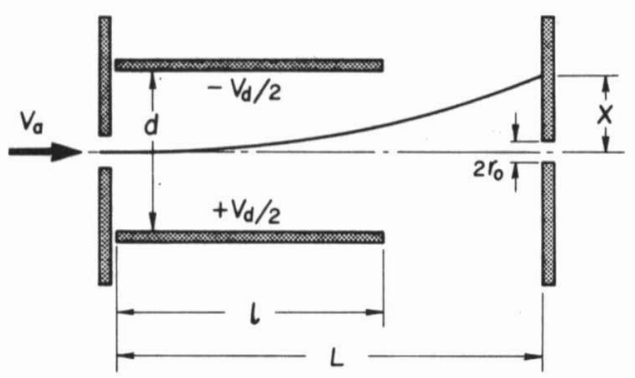

Fig. 1. Schema of the deflection system for neasuring ion beani profiles.

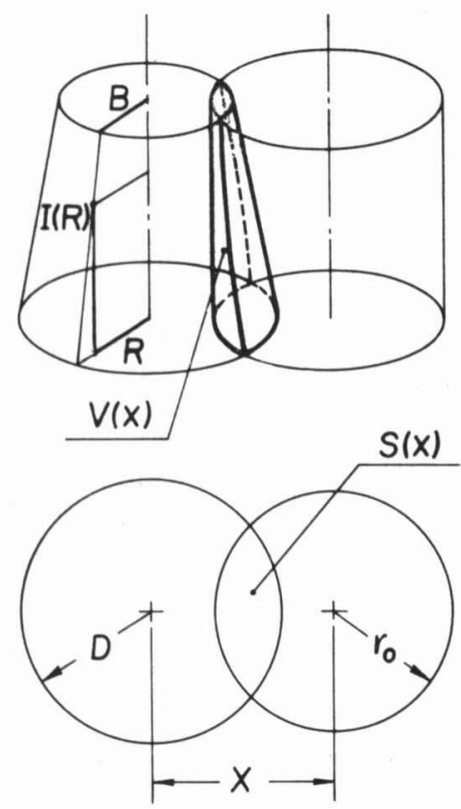

Fig. 2. Ciennetry for calculating $V(x)$ curves.

$I(X=0)$, are assigned to the original beam profile.

Following questions may arise about the method. First, to what extent is the value of $E_{\mathrm{d}}$ given by Eq. (2) affected by uniformity of the defelection field and the fringing field? Second, to what extent is the actual beam profile approximated by Eq. (3)? The most straightforward method to answer the questions is to directly determine the actual beam profile with a very small detector hole and to compare the profile with that obtained by the method I.

We have experimentally recognized that the method I can reproduce the actual beam profile with sufficient accuracy. In the following are reported the results.

In the apparatus used in this work, a plate equipped with two detector holes, $20 \mu \mathrm{m}$ and $1 \mathrm{~mm}$ in diameter, could be moved by a micrometer (see Fig. 3). Ion beam intensity was measured by an electron multiplier, Bendix M308. Two kinds of deflection electrode shapes were used for the deflection system $E_{1}$, one being thick-brimmed (system A) and the other being plane (system B), as shown in Fig. 4.

Three methods were employed in the experiments for determining the profiles.

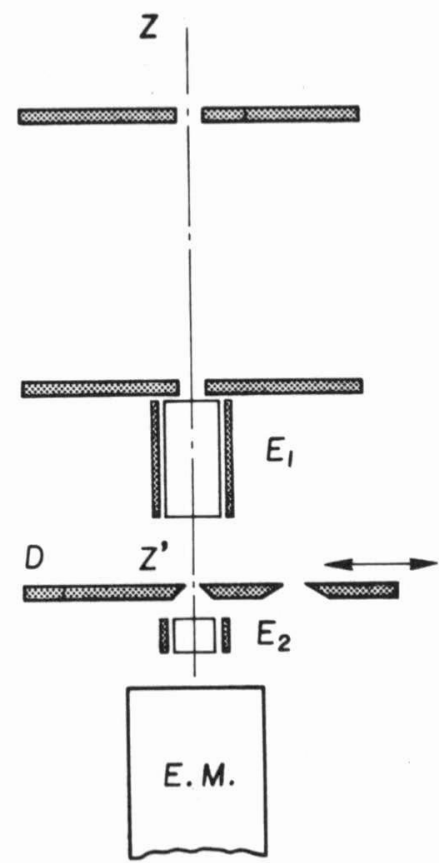

Fig. 3. Schema of the experimental apparatus; $E_{1}$ is the deflection system for measuring ion beam profiles, $E_{2}$ the deflection system for sweeping the cathode surface of the electron multiplier, E. M., with a thin ion beam, $\mathrm{D}$ a movable plate equipped with detector holes, and $\mathrm{ZZ}^{\prime}$ the axis of beam defining apertures. 


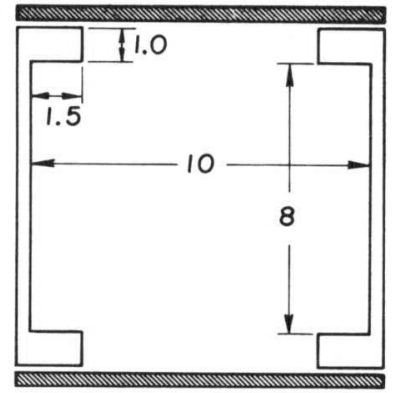

A

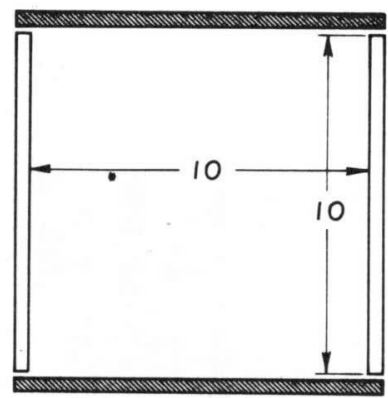

B

Fig. 4. Two kinds of deflection electrode shapes used in this work. Electrodes shown by the hatched areas are always grounded.

The first method is the method I using the detector hole of $1 \mathrm{~mm}$ in diameter. The second method is to determine the beam diameter by the deflection method with the detector hole of $20 \mu \mathrm{m}$ in diameter located on the axis, $Z Z^{\prime}$, of the beam defining apertures (method II). The true beam diameter and profile will be obtained by moving the $20 \mu \mathrm{m}$ hole across the beam (method III). Comparing the results obtained in the latter two experiments enables one to decide whether the assumption of Eq. (1) is proper or not.

Equation (2) assumes a uniform field distribution between the two facing electrodes of the deflection system. This, however, is not always true in a finite plane electrode system. One must, consequently, find an actual distribution or use such an electrode shape that produces a distribution closest to the ideal one given by Eq. (2). Comparison of the results obtained by the method II using the systems $\mathrm{A}$ and $\mathrm{B}$ with those of the method III will decide which of the electrode systems provides a deflection field in better approximation to the ideal field given by Eq. (2). Another comparison of the beam profile obtained by the method I using the $1 \mathrm{~mm}$ hole with the true profile obtained by the method III will enable one to know the validity of the procedure (2) described above.

The results of the experiments are shown in Fig. 5, according to which the beam diameter obtained by the method II using the system $\mathrm{A}$ is in excellent agreement with the value obtained by the method III. $\mathrm{W}$ ith the system $\mathrm{B}$, on the other hand, the method II resulted in an apparent beam diameter $20 \%$ greater than the method III did. This discrepancy was considered to be resulted from nonuniformity of the deflection field in the

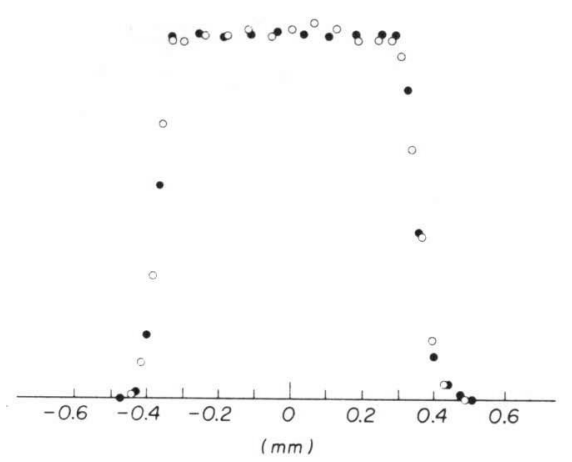

Fig. 5. Comparison of the results obtained by moving the $20 \mu \mathrm{m} \phi$ hole (open circles) with those obtained by the deflection method using the $20 \mu \mathrm{m} \phi$ hole (solid circles). 


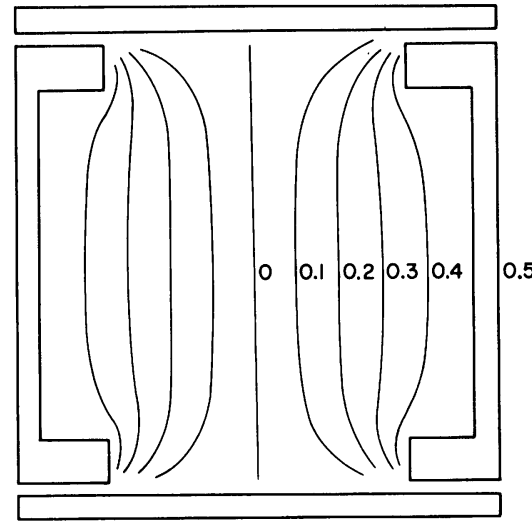

(A)

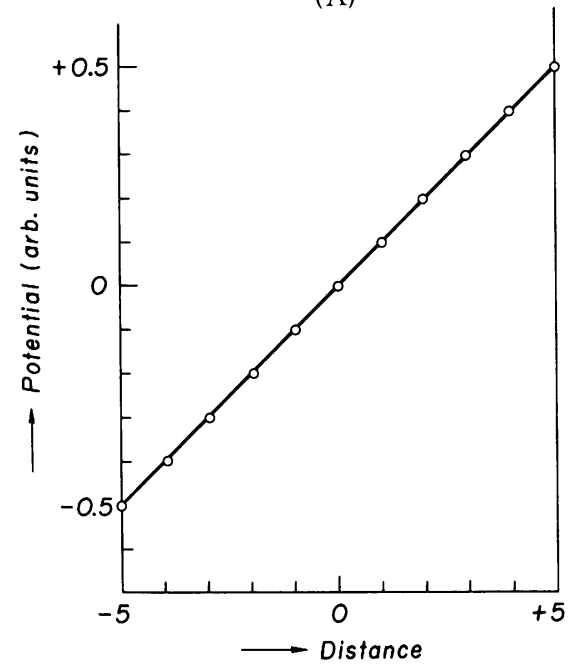

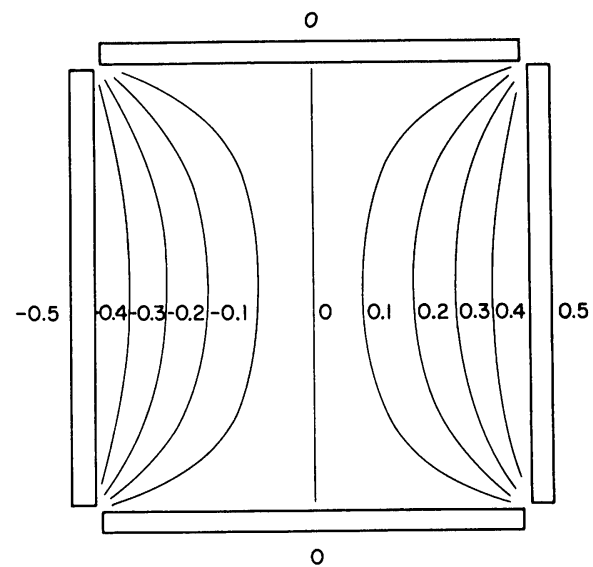

(B)

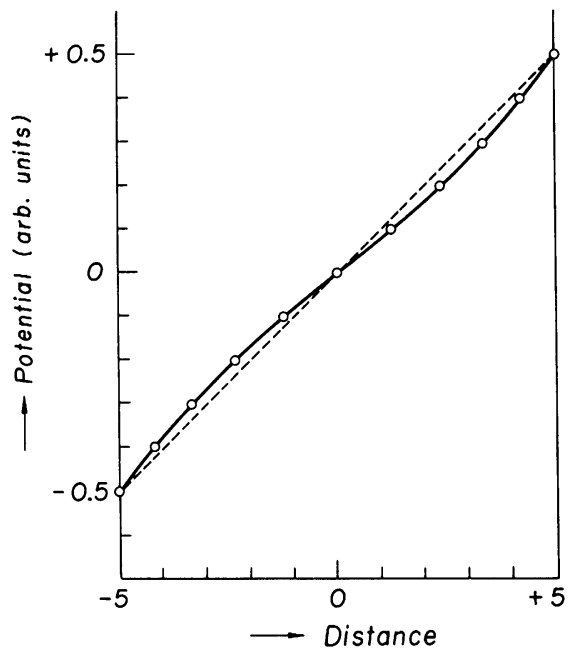

Fig. 6. Field distributions between the deflecting electrodes obtained on conducting papers. The dashed line represents the field gradient given by Eq. (2).

system B. The field distribution in the deflection system obtained with a conducting paper is given in Fig. 6, which clearly shows that in the system $B$ the field is $20 \%$ weaker than the value calculated by Eq. (2), thus resulting in the apparent beam diameter greater than the true value. The result mentioned above also suggests that the fringing field can be safely ignored in the electrode arrangement used in this work. This was also confirmed by the numerical calculation of the displacement $X$ along the fringing field curve shown in Fig. 7, which was also obtained on a conducting paper; the value of $X$ thus calculated was greater by only $2 \%$ than the value given by Eqs. (1) and (2).

Apparent intensity distribution across the beam measured by the method I with the $1 \mathrm{~mm}$ hole is shown in Fig. 8 . The original beam profile derived by deconvolution of this apparent intensity curve is of a trapezoid with the base $2 D=0.85$ and the plateau $2 B=0.65$, both in units of the hole diameter $2 r_{0}=1 \mathrm{~mm}$, as shown in Fig. 9. The solid curve in the figure is 

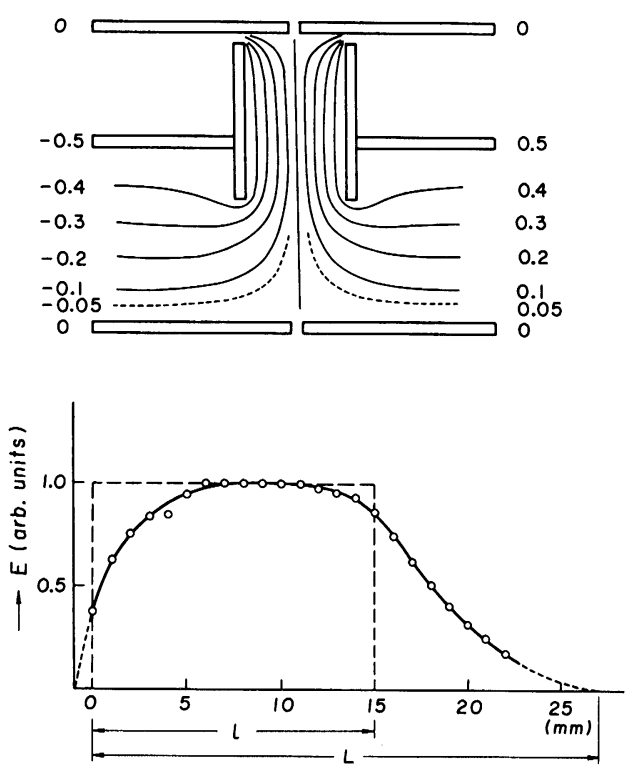

Fig. 7. Fringing field distribution obtained on a conducting paper.

the beam profile obtained by the method II using the $20 \mu \mathrm{m}$ hole and the dashed curve represents the trapezoidal profile just described; both curves are in excellent agreement. This leads to the conclusion that the profile determination by the procedure (2) described early in this arti-

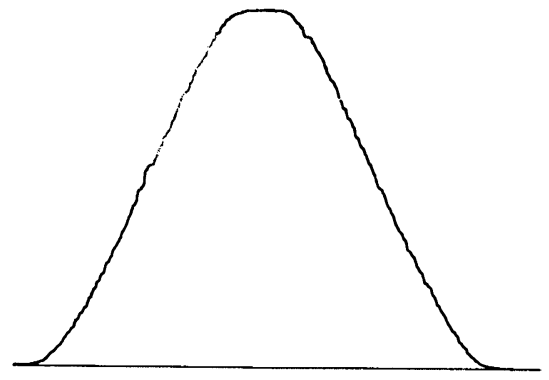

Fig. 8. Apparent intensity distribution across the beam obtained by the deflection method using the $1 \mathrm{~mm} \phi$ hole. cle gives a correct result. It may also be said that a trapezoidal beam profile which has been frequently assumed is quite a realistic one. ${ }^{8}$

It was found in the course of this work that the following cares are necessary if the intensity of an ion beam of a very small diameter (less than about $100 \mu \mathrm{m}$ ) is measured with an electron multiplier. The first is the existence of the electron suppressor grid of the multiplier. As this grid is usually made of fine wires of a few tens $\mu \mathrm{m}$, it prevents a part of the beam from reaching the cathode in some cases. Figure 10 shows the beam intensity curves $I(X)$ obtained by the method II. Periodical variation in the curves is considered to be caused by the interference of the grid. We replaced, therefore, the wire grid by a plate with a small open window in this work. This resulted in the smooth curves, as expected. Next, one should take note of uniformity of the ionelectron conversion gain over the cathode surface. Figure 11 shows the results of sweeping the surface with an ion beam of $20 \mu \mathrm{m}$ in diameter deflected by the system

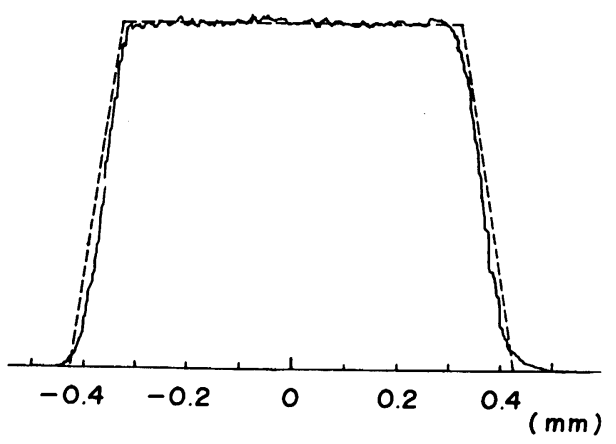

Fig. 9. Comparison of the beam profile obtained by the deflection method using the $20 \mu \mathrm{m} \phi$ hole and the deflecting electrode system A (solid curve) with the trapezoidal profile whose base is 0.85 $\mathrm{mm}$ and plateau $0.65 \mathrm{~mm}$ (dashed curve). 


\section{H. Inouye and $K$. Niurao}

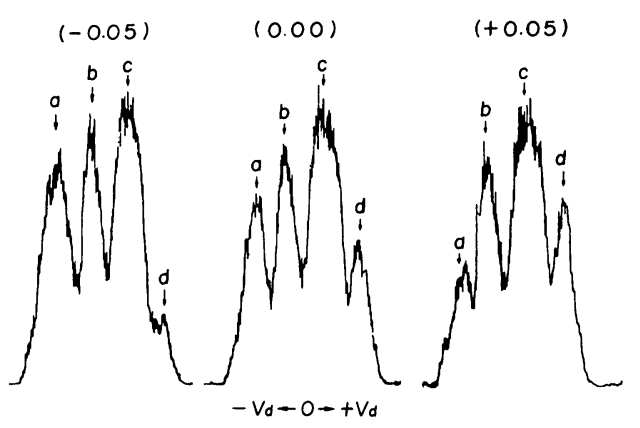

Fig. 10. Effect of the electron suppressor grid of the electron multiplier on the apparent intensity distribution curve. Numbers in parentheses represent the locations of the detector hole (in units of $\mathrm{mm}$ ). Slight displacement of the detector hole causes remarkable changes in the heights of the peaks marked with a and d. Valleys are caused by the grid which prevents a part of the beam from reaching the cathode of the multiplier.

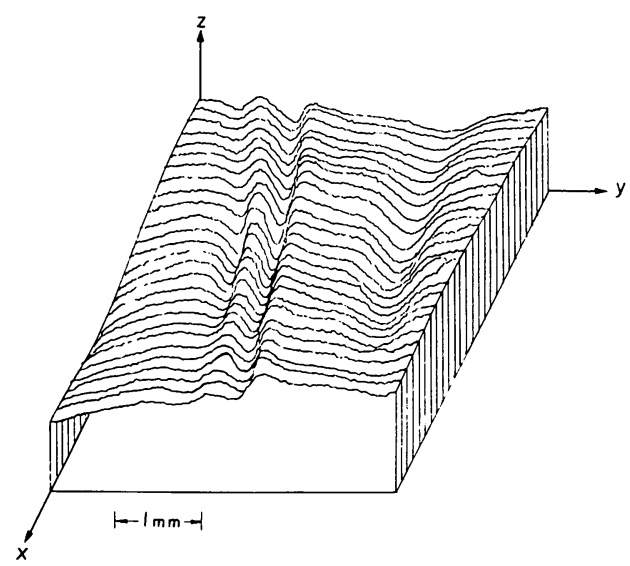

Fig. 11. Variation in the ion-electron conversion gain over the cathode surface of the electron multiplier.

$\mathrm{E}_{2}$ shown in Fig. 3. One can clearly recognize the existence of gain variation over the surface. Although the non-uniformity of the gain over the surface will be averaged in the case of a beam of large diameter, it will affect significantly the result of the intensity measurement in the case of a thin beam. Sufficient care must be exercised so as to make the beam strike an area having uniform gain.

In summary, the following has been experimentally confirmed. (1) Displacement of the beam by a transverse electric field can be correctly calculated by Eqs.(1) and (2), when the deflecting electrode system A shown in Fig. 4 is used for the determination; (2) in the electrode arrangement used in this work the fringing field does not seem to significantly affect the displacement; (3) the original beam profile can be derived with sufficient accuracy by comparing the apparent intensity distribution curve $I(X)$ with the curve calculated by Eq.(3), $V(X)$; and (4) cares are necessary about uniformity of the gain over the cathode of an electron multiplier.

\section{References}

1) J. B. Taylor, Phys. Rev., 35, 375 (1930).

2) L. Jacob, Phil. Mag., 28, 81 (1939).

3) H. Inouye, A. Takagi, and K. Inaoka, Sci. Rep. Res. Inst. Tohoku Univ., A 19, 195 (1967); H. Inouye and A. J. H. Boerboom, J. Phys., E 1, 484 (1968).

4) B. A. Probyn, J. Phys., D 1, 457 (1968).

5) R. B. Fair, J. Phys., E 4, 35 (1971).

6) R. Akselsson and T. B. Johansson, Nucl. Instr. Meth., 91, 663 (1971).

7) J. K. Kleiwer, D. D. Sharma, and T. A. Dardis, Nucl. Instr. Meth., 97, 611 (1971).

8) H. Inouye and S. Kita, J. Chem. Phys., 56, 4877 (1972); ibid., 57, 1301 (1972). 